\title{
OGHReS: Large-scale filaments in the outer Galaxy ${ }^{\star}$
}

\author{
D. Colombo ${ }^{1}$, C. König ${ }^{1}$, J. S. Urquhart ${ }^{2}$, F. Wyrowski ${ }^{1}$, M. Mattern ${ }^{3}$, K. M. Menten ${ }^{1}$, M.-Y. Lee ${ }^{4}$, J. Brand ${ }^{5}$, \\ M. Wienen ${ }^{1}$, P. Mazumdar ${ }^{1}$, F. Schuller ${ }^{1,6}$, and S. Leurini ${ }^{7}$ \\ 1 Max-Planck-Institut für Radioastronomie, Auf dem Hügel 69, 53121 Bonn, Germany \\ e-mail: dcolombo@mpifr-bonn.mpg.de \\ 2 Centre for Astrophysics and Planetary Science, University of Kent, Canterbury CT2 7NH, UK \\ 3 Commissariat à l'énergie atomique et aux énergies alternatives, 91191 Gif-sur-Yvette, Saclay, France \\ 4 Korea Astronomy and Space Science Institute, 776 Daedeok-daero, Yuseong-gu, Daejeon 34055, Republic of Korea \\ 5 INAF - Istituto di Radioastronomia and Italian ALMA Regional Centre, via P. Gobetti 101, 40129 Bologna, Italy \\ ${ }^{6}$ Leibniz-Institut für Astrophysik Potsdam (AIP), An der Sternwarte 16, 14482 Potsdam, Germany \\ 7 INAF - Osservatorio Astronomico di Cagliari, Via della Scienza 5, 09047 Selargius, CA, Italy
}

Received 8 September 2021 / Accepted 27 October 2021

\begin{abstract}
Filaments are a ubiquitous morphological feature of the molecular interstellar medium and are identified as sites of star formation. In recent years, more than 100 large-scale filaments (with a length $>10 \mathrm{pc}$ ) have been observed in the inner Milky Way. As they appear linked to Galactic dynamics, studying those structures represents an opportunity to link kiloparsec-scale phenomena to the physics of star formation, which operates on much smaller scales. In this Letter, we use newly acquired Outer Galaxy High Resolution Survey (OGHReS) ${ }^{12} \mathrm{CO}(2-1)$ data to demonstrate that a significant number of large-scale filaments are present in the outer Galaxy as well. The 37 filaments identified appear tightly associated with inter-arm regions. In addition, their masses and linear masses are, on average, one order of magnitude lower than similar-sized molecular filaments located in the inner Galaxy, showing that Milky Way dynamics is able to create very elongated features in spite of the lower gas supply in the Galactic outskirts.
\end{abstract}

Key words. ISM: molecules - ISM: clouds - evolution - ISM: structure - local insterstellar matter - galaxies: ISM

\section{Introduction}

Since the early observations with Herschel (André et al. 2010), it became evident that the molecular interstellar medium (ISM) has a preference to organise itself in filaments. Filaments are ubiquitous in the Galaxy and are found on a large variety of scales. Networks of infrared dust extinction filamentary features (indication high column densities) constitute molecular clouds (e.g., André et al. 2010; Men'shchikov et al. 2010; Arzoumanian et al. 2011; Schneider et al. 2012; Li et al. 2016; Schisano et al. 2020). Elongated structures within clouds are also observed in emission (Panopoulou et al. 2014; Suri et al. 2019). Clouds themselves are mostly elongated structures (Duarte-Cabral et al. 2021, Neralwar et al., in prep.). Additionally, bundles of smaller scale 'fibers' have been found within those filaments (Hacar et al. 2013, 2016; Henshaw et al. 2016). Extremely long (even longer than $100 \mathrm{pc}$ ) and velocity coherent large-scale filaments (LSFs) have been observed in the Milky Way as part of spiral arms or inter-arm regions (Jackson et al. 2010; Goodman et al. 2014; Ragan et al. 2014; Zucker et al. 2015, 2018; Abreu-Vicente et al. 2016; Li et al. 2016; Du et al. 2017; Lin et al. 2020; Schisano et al. 2020). As the physics of filaments appears tightly connected to star formation (e.g., André et al. 2010, 2014; Elia et al. 2013), a great deal of studies have been put forward to explain the origin and evolution

\footnotetext{
* Datacubes are only available at the CDS via anonymous ftp to cdsarc.u-strasbg.fr (130.79.128.5) or via http://cdsarc. u-strasbg.fr/viz-bin/cat/J/A+A/655/L2
}

of those structures. Filaments have been linked to ISM turbulence (Padoan et al. 2001), and also to cloud-cloud collisions, expanding shells, and magnetic fields (e.g., Hartmann \& Burkert 2007; Heitsch et al. 2008; Molinari et al. 2014). LSFs instead appear to be formed by large-scale phenomena such as spiral arm shocks, galactic shear, and supernovae (SNe) feedback. Simulations have found LSFs in both spiral arms and interarm regions (e.g., Dobbs \& Pringle 2013; Smith et al. 2014, 2020). However, the simulations of Smith et al. (2014) predicted that LSFs originate within spiral arms, supporting the Galactic 'bone' paradigm (Goodman et al. 2014; Zucker et al. 2015), while the work by Duarte-Cabral \& Dobbs (2016), whose simulations also include $\mathrm{SNe}$ feedback and gas self-gravity, indicate that LSFs originate exclusively by the intense shear of the interarm regions. Upon entry in the spiral arms, those filaments lose their elongated morphology and merge to become large cloud complexes (Duarte-Cabral \& Dobbs 2017). More recently, the work of Smith et al. (2020), instead, simulated LSFs in both spiral arms and inter-arm regions. They also found that those structures tend to be longer than filaments that formed under the action of SNe feedback, which in turn have large velocity gradients and might be transient features of the ISM.

In this Letter we continue the search of LSFs in the outskirts of the Milky Way. The outer Galaxy presents different characteristics compared to the inner Galaxy, in terms of the radiation field, metallicity, HI density, and gas-to-dust ratio values (see Appendix A for more details). Therefore, it represents an opportunity to study the properties of the LSFs in 
largely unexplored environmental conditions. Using ${ }^{12} \mathrm{CO}(2-1)$ data from the OGHReS science demonstration phase (SD1; see Appendix B.1 and König et al., in prep. for further details), we identified tens of outer Galaxy LSFs (hereafter OGLSFs) and compared their properties with similar-scale objects observed in the inner Milky Way.

\section{Results}

To search for filaments in OGHReS data (between $250<l<$ $280 \mathrm{deg}$ ), we masked the data considering only the high signalto-noise ratio $(\mathrm{S} / \mathrm{N})$ emission and we used a dendrogram analysis (Rosolowsky et al. 2008) to isolate coherent structures (see Appendix B.3 for more details). As OGLSFs, we considered all of the 'trunks' of the dendrogram with a length $>10 \mathrm{pc}$, which is a clear filamentary morphology (or a typical aspect ration $A R>10)$, and observed at $V_{\mathrm{LSR}}>40 \mathrm{~km} \mathrm{~s}^{-1}$. Lengths and $A R$ were measured through the 'medial axis analysis' and RadFil (Zucker et al. 2018). Using this method, we identified 37 OGLSFs, which is over a factor of 7 more than the LSFs currently identified in the outer Galaxy (Du et al. 2017). However, while we focused on all filamentary structures with a length $>10 \mathrm{pc}$, the work of Du et al. (2017) considers only the longest structures (with a length $>55 \mathrm{pc}$ ). In this aspect, we have a comparable number of filaments (ten objects with a length $\sim 40-144 \mathrm{pc}$; see Fig. 1 and Appendix C), despite having searched for them in roughly a third of the Galactic area surveyed by Du et al. (2017). Additionally, our search focuses on only the most prominent structures and does not consider all LSFs present in OGHReS SD1 data as we have included only connected and isolated structures at $V_{\mathrm{LSR}}>40 \mathrm{~km} \mathrm{~s}^{-1}$. Indeed, we have also observed LSFs within the 'local' gas (at $\left.V_{\mathrm{LSR}} \sim 0 \mathrm{~km} \mathrm{~s}^{-1}\right)$, objects with a length and $A R$ below our assumed thresholds, and velocity coherent fragments of filaments that dendrograms alone are not able to merge into a single structure. The 37 OGLSFs represent a lower limit of the actual number of filaments present in the survey. We might expect to find at least $\sim 120$ similar properties of LSFs in the full 100 $\operatorname{deg}^{2}$ span by the full OGHReS data, once the survey is completed. A full catalogue of all filamentary structures present within OGHReS data will be presented in a future work. This would be comparable to the current number of LSFs observed in the inner Galaxy (approximately 70 objects), whose properties we use here for comparison. Those inner Galaxy large-scale filaments (hereafter IGLSFs) have been found by different studies and have been named according to their appearance or identification methods (see Appendix B.2 for more details). The properties of the IGLSFs that we use here have been re-elaborated by Zucker et al. (2018) and Zhang et al. (2019), with the addition of the filaments from Mattern et al. (2018a).

Integrated intensity maps of the ${ }^{12} \mathrm{CO}(2-1)$ emission from the longest OGLSFs (with a length $>40 \mathrm{pc}$ ) are compiled in Fig 1, which illustrates the complexity of the LSFs observed. All selected LSFs are velocity coherent structures (where the median of the velocity dispersion distribution is $\sim 1 \mathrm{~km} \mathrm{~s}^{-1}$ ) which might indicate that those filaments are young or quiescent.

The location of the OGLSFs across the Milky Way disc is shown in Fig. 2. IGLSFs are observed at heliocentric distances $1.3-11.6 \mathrm{kpc}$, similar to OGLSFs that show distances $4.2-10.6 \mathrm{kpc}$. Nevertheless, IGLSFs are distributed within $R_{\mathrm{Gal}}=1-7 \mathrm{kpc}$, while our OGLSFs are further away from the Galactic centre and show $R_{\mathrm{Gal}}=9-13 \mathrm{kpc}$. Despite the giant molecular filaments (GMFs) class, for which two-thirds of the objects are found in the inter-arm region (Zucker et al. 2018) (see also Ragan et al. 2014; Abreu-Vicente et al. 2016), other IGLSF categories appear closer to or even within spiral arms (Jackson et al. 2010; Goodman et al. 2014; Zucker et al. 2015, 2018; Wang et al. 2015, 2016). Instead, OGLSFs seem to have all inter-arm features, as only three of them have a velocity offset $(\Delta V)$ with respect to the closest spiral arm (the Perseus arm, assuming the Taylor \& Cordes 1993 spiral arm models, as adopted in Urquhart et al. 2021) $<10 \mathrm{~km} \mathrm{~s}^{-1}$. The others are all kinematically far from the Perseus arm with a median $\Delta V=$ $30.2 \mathrm{~km} \mathrm{~s}^{-1}$ (and a median absolute deviation of $8.7 \mathrm{~km} \mathrm{~s}^{-1}$; see also Table C.1).

A comparison of some of the properties of the LSFs observed in the inner and outer Galaxy is shown in Fig. 3. Here, we only compare properties that are also available from the SEDIGISM catalogue. The OGLSFs show some of the smallest values of mass within the full sample, ranging from $\sim 10^{2}-7 \times 10^{4} M_{\odot}$, while most of the inner Galaxy structures show masses $\sim 3 \times$ $10^{3}-5 \times 10^{5} M_{\odot}$. An exception are the GMFs with masses of $\sim 2.5 \times 10^{4}-2.5 \times 10^{6} M_{\odot}$. By construction, all LSFs have a minimum length $\sim 10 \mathrm{pc}$. However, Herschel filaments show a minimum length of $42 \mathrm{pc}$. The GMFs are the longest filaments, on average, in the sample having lengths from $56-270 \mathrm{pc}$, with a median of 94 pc (for Zucker et al. 2018 estimations, while considering Zhang et al. 2019 measurements, GMFs show a minimum length $\sim 31 \mathrm{pc}$ ). Indeed, only the longest structures in the other categories reach the median length of the GMFs. Due to their low mass content, the OGLSFs show some of the lowest median values of linear mass (mass over the length of the structures) between the full sample, having $\sim 10-600 M_{\odot} \mathrm{pc}^{-1}$, while for the IGLSFs we observe linear masses $\sim 86-9200 M_{\odot} \mathrm{pc}^{-1}$, where the largest linear mass is again observed for the GMFs (for more details regarding the mass estimation reliability across the different works, see Appendix B.4).

\section{Discussion and conclusions}

In the previous section, we observed several properties that distinguish the OGLSFs from the IGLSFs. In particular, many IGLSFs are associated with the spiral arms, while all the OGLSFs appear as inter-arm objects. In addition to having similar lengths as the LSFs in the inner Galaxy (whose lower limit is set by construction), OGLSFs are in general less massive, resulting in much lower linear masses compared to IGLSFs. Taken together, these might indicate a different origin, and possibly evolution, for the filaments observed in the spiral arms versus inter-arm regions, and inner versus outer Galaxy. The analysis of Zucker et al. (2019) performed on Smith et al. (2020) simulations showed that spiral arm filaments tend to have masses and linear masses up to $5 \times$ higher compared to similar-length interarm objects, indicating that the Galactic environment can have a significant impact on their properties. Even if this work is based on very long filaments $(>100 \mathrm{pc})$, its conclusions are in line with our results. Nevertheless, from an observational point of view, some caveats need to be considered. First of all, the exact morphology of the Milky Way is not well known (Dobbs \& Baba 2014), especially in its outskirts (Koo et al. 2017), which renders the exact localisation of the filaments with respect to the spiral structure challenging. Indeed, only a third of the IGLSFs analysed by Zucker et al. (2018) can be spatially and kinematically associated with spiral arms. However, this result does not include all objects in Zucker et al. (2018), and it is modeldependent (e.g., Zucker et al. 2015). Additionally, distance measurements in the inner Galaxy are challenging due to a number of effects, such as the kinematic distance ambiguity (KDA) and 

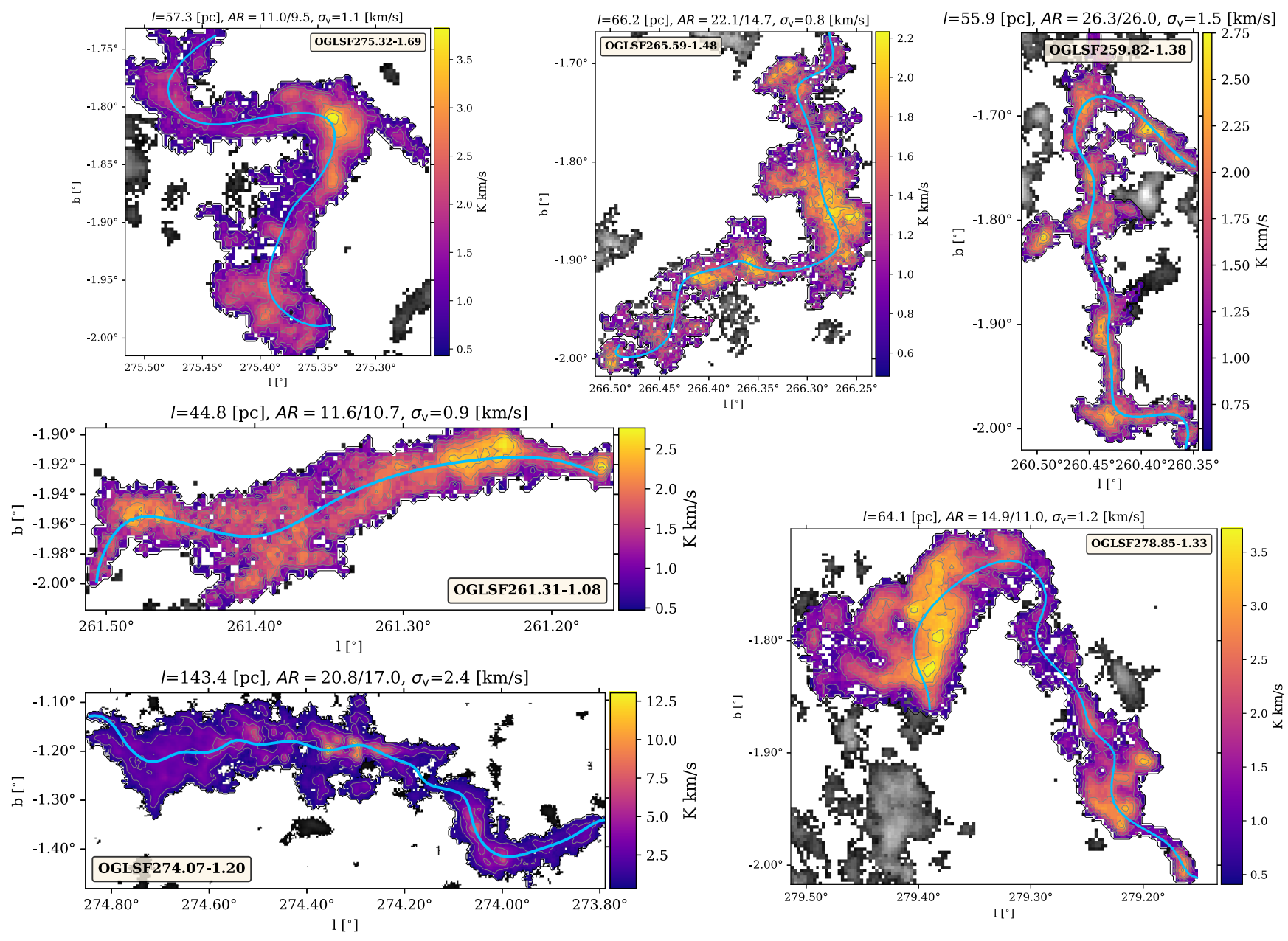

Fig. 1. Integrated intensity maps of the ${ }^{12} \mathrm{CO}(2-1)$ emission from some of the most prominent outer Galaxy LSFs identified (in colour) within the structure masked data defined by the dendrogram. In grey-scale, the emission across the line-of-sight that does not belong to the structure is shown. The cyan line displays the filament spine. In the box, the structure name is indicated. In the panel titles, the length $(l)$, the aspect ratio $\left(A R=\right.$ length/width, with the width inferred from the medial axis method/RadFil), and the velocity dispersion $\left(\sigma_{\mathrm{v}}\right.$, calculated as the intensityweighted second moment of velocity) of the structures are shown. The velocity range spanned by the structures is reported in Table C.1 as 5th and 95th percentiles of the LSF velocity distribution.

spiral arm velocity crowding. The outer Galaxy does not suffer from these issues, therefore kinematic distance-dependent properties of the OGLSFs can be considered to be more robust than for their inner Milky Way counterparts. Nevertheless, for sources observable from the northern hemisphere, trigonometric parallax distances determined for radio wavelength maser sources with very long baseline interferometry (VLBI) are used to 'anchor' the locations and dimensions of spiral arms in the first and second Galactic quadrants (and part of the third Reid et al. 2019). So far, the distances of only very few southern hemisphere have been measured with VLBI (Krishnan et al. 2015, 2017) and none of them are within the longitude range considered here $(250<l<280 \mathrm{deg})$.

It is interesting to note that (considering the analysis of Zucker et al. 2018) two-thirds of the GMFs appear to be interarm features, as our OGLSFs. However, the property distributions of GMFs and OGLSFs are the most discrepant within the sample. Some of these differences might be intrinsically methodological. For example, the GMF category contains the longest objects in the sample, while only a few OGLSFs reach the length of an average GMF. GMFs have been firstly identified in extinction using GLIMPSE data (Benjamin et al. 2003); subsequently, their velocity coherence has been verified with GRS
${ }^{13} \mathrm{CO}(1-0)$ data (Jackson et al. 2006). We used the GMF properties presented in Zucker et al. (2018) and Zhang et al. 2019. In our case, instead, all of those operations were made via emission data. Our searching method does not allow us to connect fragments of a filament, but only finds velocity coherent regions that, as a whole, stand out above the noise level. Those velocity coherent fragments might be abundant in our data given the low brightness of the gas emission in the outer Galaxy and the limited sensitivity. Therefore our catalogue might be biased towards shorter filaments. Considering that various methods appear to provide similar mass estimates for IGLSFs and OGLSFs (see Appendix B.4), mass differences between GMFs (or generally also of the IGLSFs) and OGLSFs might be due to less molecular gas supply in the outer Galaxy compared to the inner Galaxy. The molecular gas distribution across the disc of the Milky Way varies significantly with the Galactocentric radius. The molecular gas mass surface density decreases by a factor of a few moving from the inner to the outer Galaxy (see Heyer \& Dame 2015, their Fig. 7). For instance, for similar large-scale effects operating in the inter-arm regions across the Milky Way, there might simply not be enough gas available to build up filaments as massive as GMFs (and with similar linear masses) in the outer Galaxy. Indeed, the OGLSFs observed here have a peculiar 


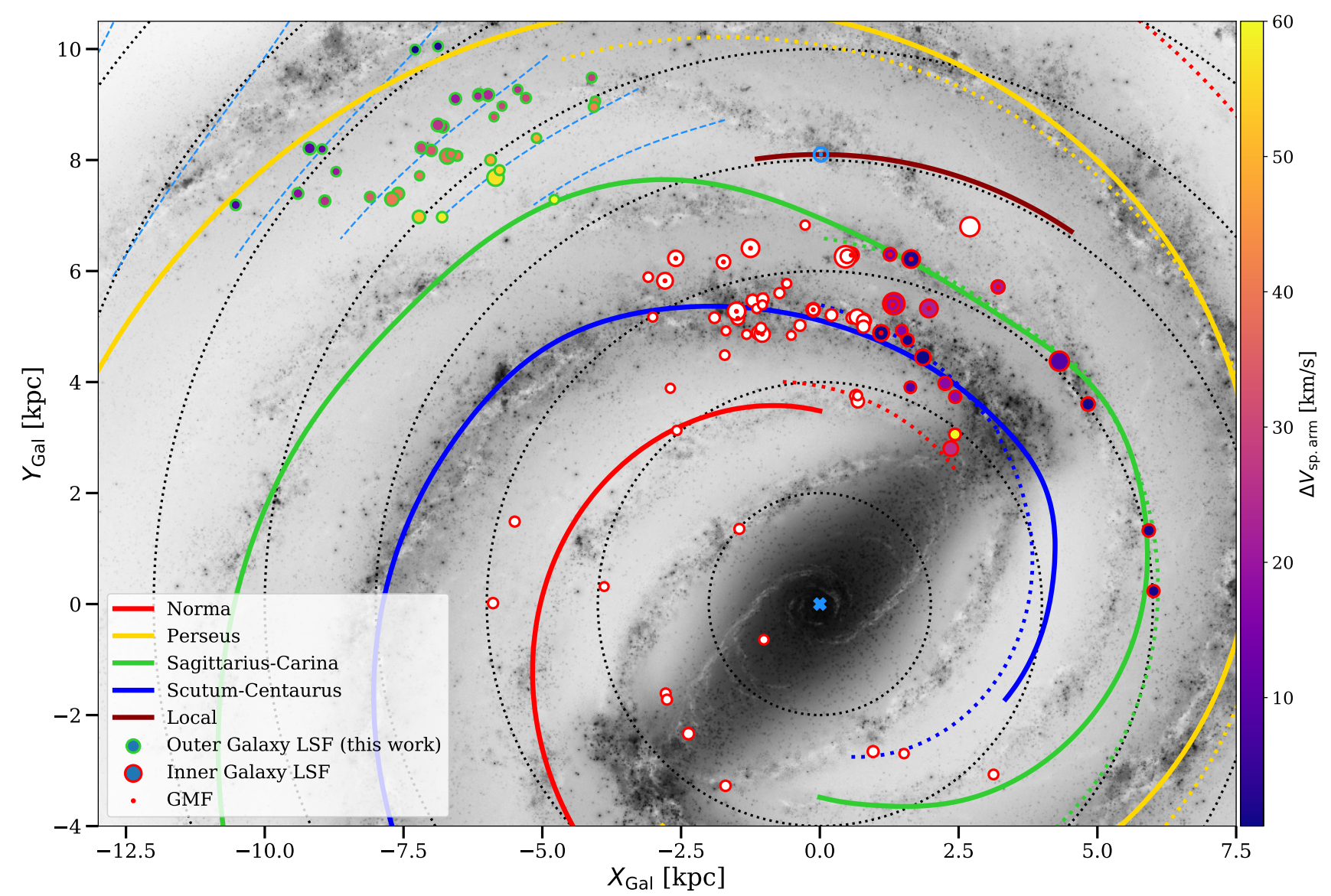

Fig. 2. Location of the large-scale filaments (LSFs) across the Milky Way disc. Inner Galaxy LSFs and outer Galaxy LSFs are indicated with red and green contoured circles, respectively. The size of the circles is proportional to the filament lengths. The inner colours of markers show the velocity offset $\left(\Delta V_{\text {sp.arm }}\right)$ with respect to the closest spiral arm (see the colour scale on the right). White-filled markers are for inner Galaxy LSFs where this quantity is not provided by their original catalogues. Inner Galaxy giant molecular filaments (GMFs) are highlighted with red dots. Coloured full lines mark the position of the spiral arms following Taylor \& Cordes (1993) models, as adapted in Urquhart et al. (2021). The 'Local' arm (or spur) parameters are taken from Reid et al. (2019). Dotted coloured lines show the correspondent spiral arm loci from Reid et al. (2016), used to define the spiral arm associations in the inner Galaxy. Galactocentric circles drawn with black dotted lines are spaced $2 \mathrm{kpc}$ apart. Cyan dashed arcs show loci with equal velocity between $20-120 \mathrm{~km} \mathrm{~s}^{-1}$ (from the Galactic centre outwards) and spaced $20 \mathrm{~km} \mathrm{~s}$ apart. The cyan ' $\mathrm{X}$ ' indicates the Galactic centre and ' $\odot$ ' the position of Sun. The figure was produced using the Mw-PLOT package (available at https://milkyway-plot.readthedocs.io/en/latest/).
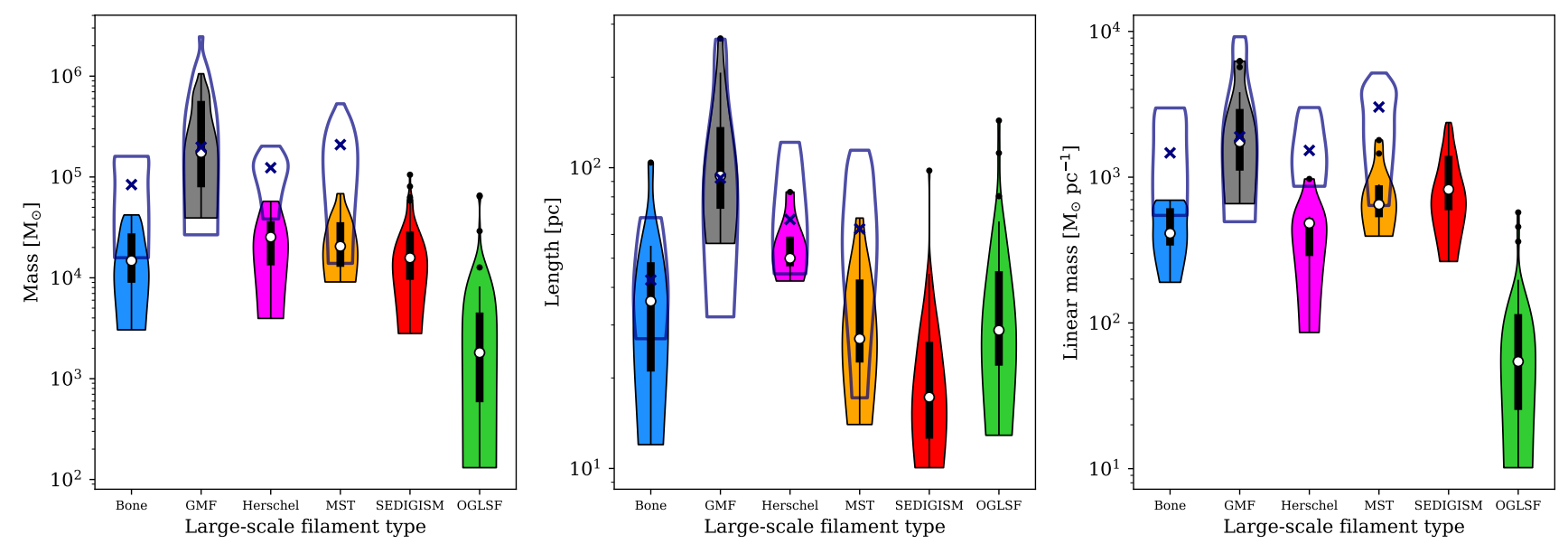

Fig. 3. Violin- (colours) and box-plot (black) representations of the distributions of mass (left), length (middle), and linear mass (right) of Galactic LSFs. Following Zucker et al. (2018), IGLSFs are divided into Milky Way 'bones' (blue), GMFs (grey), Herschel filaments (magenta), minimum spanning tree (MST) filaments (yellow). IGLSFs identified from the Structure, Excitation, and Dynamics of the Inner Galactic InterStellar Medium (SEDIGISM, Schuller et al. 2017) survey data are in red (from Mattern et al. 2018b). OGLSFs, observed within our data, are in green. More information on IGLSFs are reported in Appendix B.2. The white circles show the median for each property and sub-sample. For comparison, the transparent violins show the measurements of Zhang et al. (2019) for the same category of structures, where the ' $x$ ' indicates the median of the samples. 
filamentary morphology, but their masses are in-line with the typical mass of clouds in the outer Galaxy $\left(<10^{4} M_{\odot}\right.$, Heyer et al. 2001, but see also Miville-Deschênes et al. 2017), whose maximum value appears to be an order of magnitude lower compared to the clouds in the Solar circle (see Brand \& Wouterloot 1995, their Fig. 8). The rapid decrement of the molecular gas mass surface density with the distance might also be the reason why our OGLSFs are almost all observed in the inter-arm region. At the longitudes spanned by our data, the lines-of-sight cross two spiral arms, the Perseus and the Outer arms. However, as they are both located at far distances $\left(R_{\mathrm{Gal}}>12 \mathrm{kpc}\right)$, they appear faint and patchy in our data, mostly encompassing low S/N flux (see König et al., in prep.). Therefore, OGHReS SD1 imaged mostly emission originating from the inter-arm region. Only the longest (143.4 pc) filament observed within our sample, OGLSF274.07-1.20 (see Fig. 1), has properties comparable with inner Galaxy filaments. Additionally, similar to the IGLSFs (e.g., Zhang et al. 2019), it is actively forming stars and embedding a SN remnant (Duncan et al. 1996) and several HII regions (e.g. Haverkorn et al. 2006; Culverhouse et al. 2011). As SNe feedback appears to be the main mechanism responsible for filament dissipation (see simulations by Smith et al. 2020), this structure might be transient in nature.

In summary, we have assembled the largest catalogue, to date, of emission-selected outer Galaxy large-scale filaments (with a length $\gtrsim 10 \mathrm{pc}$ and $A R \gtrsim 10$ ), consisting of 37 velocity coherent objects located at $R_{\mathrm{Gal}}>9 \mathrm{kpc}$. Almost all OGLSFs appear to be part of inter-arm regions and have masses and linear masses, on average, one order of magnitude lower than other LSFs observed in the inner Galaxy. The completion of the OGHReS project, with $70 \mathrm{deg}^{2}$ more molecular gas emission mapped, will provide access to more than 100 similar objects in the outer Milky Way and shed new light on how galactic dynamics shapes molecular clouds (and ultimately star formation) in a largely unexplored environment of our own Galaxy.

Acknowledgements. The authors thank the anonymous referee for the feedback that helped improving the clearness of the paper. D. C. acknowledges support by the German Deutsche Forschungsgemeinschaft, DFG project number SFB956A D. C. thanks Miaomiao Zhang for the help and the useful discussion regarding the inner Galaxy filament properties. S. L. acknowledges financial support from INAF through the grant Fondi mainstream 'Heritage of the current revolution in star formation: the Star-forming filamentary Structures in our Galaxy'. This research made use of Astropy (http: //www . astropy . org), a communitydeveloped core Python package for Astronomy (Astropy Collaboration 2013, 2018); matplotlib (Hunter 2007); numpy and scipy (Virtanen et al. 2020).

\section{References}

Abreu-Vicente, J., Ragan, S., Kainulainen, J., et al. 2016, A\&A, 590, A131 André, P., Men'shchikov, A., Bontemps, S., et al. 2010, A\&A, 518, L102 André, P., Di Francesco, J., Ward-Thompson, D., et al. 2014, in Protostars and Planets VI, eds. H. Beuther, R. S. Klessen, C. P. Dullemond, \& T. Henning, 27

Arzoumanian, D., André, P., Didelon, P., et al. 2011, A\&A, 529, L6 Astropy Collaboration (Robitaille, T. P., et al.) 2013, A\&A, 558, A33 Astropy Collaboration (Price-Whelan, A. M., et al.) 2018, AJ, 156, 123 Barnes, P. J., Muller, E., Indermuehle, B., et al. 2015, ApJ, 812, 6 Benedettini, M., Traficante, A., Olmi, L., et al. 2021, A\&A, 654, A144 Benjamin, R. A., Churchwell, E., Babler, B. L., et al. 2003, PASP, 115, 953 Bloemen, J. B. G. M., Bennett, K., Bignami, G. F., et al. 1984, A\&A, 135, 12 Bolatto, A. D., Wolfire, M., \& Leroy, A. K. 2013, ARA\&A, 51, 207 Brand, J., \& Blitz, L. 1993, A\&A, 275, 67

Brand, J., \& Wouterloot, J. G. A. 1995, A\&A, 303, 851

Culverhouse, T., Ade, P., Bock, J., et al. 2011, ApJS, 195, 8
Dobbs, C., \& Baba, J. 2014, PASA, 31

Dobbs, C. L., \& Pringle, J. E. 2013, MNRAS, 432, 653

Du, X., Xu, Y., Yang, J., \& Sun, Y. 2017, ApJS, 229, 24

Duarte-Cabral, A., \& Dobbs, C. L. 2016, MNRAS, 458, 3667

Duarte-Cabral, A., \& Dobbs, C. L. 2017, MNRAS, 470, 4261

Duarte-Cabral, A., Colombo, D., Urquhart, J. S., et al. 2021, MNRAS, 500, 3027 Duncan, A. R., Stewart, R. T., Haynes, R. F., \& Jones, K. L. 1996, MNRAS, 280, 252

Elia, D., Molinari, S., Fukui, Y., et al. 2013, ApJ, 772, 45

Frerking, M. A., Langer, W. D., \& Wilson, R. W. 1982, ApJ, 262, 590 Giannetti, A., Leurini, S., König, C., et al. 2017, A\&A, 606, L12 Goodman, A. A., Alves, J., Beaumont, C. N., et al. 2014, ApJ, 797, 53 Güsten, R., Nyman, L. A., Schilke, P., et al. 2006, A\&A, 454, L13 Hacar, A., Tafalla, M., Kauffmann, J., \& Kovács, A. 2013, A\&A, 554, A55 Hacar, A., Kainulainen, J., Tafalla, M., Beuther, H., \& Alves, J. 2016, A\&A, 587, A97

Hartmann, L., \& Burkert, A. 2007, ApJ, 654, 988

Haverkorn, M., Gaensler, B. M., McClure-Griffiths, N. M., Dickey, J. M., \& Green, A. J. 2006, ApJS, 167, 230

Heitsch, F., Hartmann, L. W., \& Burkert, A. 2008, ApJ, 683, 786

Henshaw, J. D., Caselli, P., Fontani, F., et al. 2016, MNRAS, 463, 146

Heyer, M., \& Dame, T. M. 2015, ARA\&A, 53, 583

Heyer, M. H., Carpenter, J. M., \& Snell, R. L. 2001, ApJ, 551, 85

Hunter, J. D. 2007, Comput. Sci. Eng., 9, 90

Jackson, J. M., Rathborne, J. M., Shah, R. Y., et al. 2006, ApJS, 163, 145

Jackson, J. M., Finn, S. C., Chambers, E. T., Rathborne, J. M., \& Simon, R. 2010, ApJ, 719, L185

Kalberla, P. M. W., \& Kerp, J. 2009, ARA\&A, 47, 27

Koch, E. W., \& Rosolowsky, E. W. 2015, MNRAS, 452, 3435

König, C., Urquhart, J. S., Wyrowski, F., Colombo, D., \& Menten, K. M. 2021, A\&A, 645, A113

Koo, B.-C., Park, G., Kim, W.-T., et al. 2017, PASP, 129

Krishnan, V., Ellingsen, S. P., Reid, M. J., et al. 2015, ApJ, 805, 129

Krishnan, V., Ellingsen, S. P., Reid, M. J., et al. 2017, MNRAS, 465, 1095

Li, G.-X., Urquhart, J. S., Leurini, S., et al. 2016, A\&A, 591, A5

Lin, L.-H., Wang, H.-C., Su, Y., Li, C., \& Yang, J. 2020, Res. Astron. Astrophys., 20, 143

Mattern, M., Kainulainen, J., Zhang, M., \& Beuther, H. 2018a, A\&A, 616, A78

Mattern, M., Kauffmann, J., Csengeri, T., et al. 2018b, A\&A, 619, A166

Men'shchikov, A., André, P., Didelon, P., et al. 2010, A\&A, 518, L103

Miville-Deschênes, M.-A., Murray, N., \& Lee, E. J. 2017, ApJ, 834, 57

Molinari, S., Bally, J., Glover, S., et al. 2014, in Protostars and Planets VI, eds.

H. Beuther, R. S. Klessen, C. P. Dullemond, \& T. Henning, 125

Nishimura, A., Tokuda, K., Kimura, K., et al. 2015, ApJS, 216, 18

Padoan, P., Juvela, M., Goodman, A. A., \& Nordlund, A. 2001, ApJ, 553, 227

Panopoulou, G. V., Tassis, K., Goldsmith, P. F., \& Heyer, M. H. 2014, MNRAS, 444, 2507

Pitts, R. L., \& Barnes, P. J. 2021, ApJS, 256, 3

Ragan, S. E., Henning, T., Tackenberg, J., et al. 2014, A\&A, 568, A73

Reid, M. J., Menten, K. M., Brunthaler, A., et al. 2014, ApJ, 783, 130

Reid, M. J., Dame, T. M., Menten, K. M., \& Brunthaler, A. 2016, ApJ, 823, 77

Reid, M. J., Menten, K. M., Brunthaler, A., et al. 2019, ApJ, 885, 131

Rosolowsky, E., \& Leroy, A. 2006, PASP, 118, 590

Rosolowsky, E. W., Pineda, J. E., Kauffmann, J., \& Goodman, A. A. 2008, ApJ, 679, 1338

Sandstrom, K. M., Leroy, A. K., Walter, F., et al. 2013, ApJ, 777, 5

Schisano, E., Molinari, S., Elia, D., et al. 2020, MNRAS, 492, 5420

Schneider, N., Csengeri, T., Hennemann, M., et al. 2012, A\&A, 540, L11

Schuller, F., Menten, K. M., Contreras, Y., et al. 2009, A\&A, 504, 415

Schuller, F., Csengeri, T., Urquhart, J. S., et al. 2017, A\&A, 601, A124

Schuller, F., Urquhart, J. S., Csengeri, T., et al. 2021, MNRAS, 500, 3064

Smith, R. J., Glover, S. C. O., \& Klessen, R. S. 2014, MNRAS, 445, 2900

Smith, R. J., Treß, R. G., Sormani, M. C., et al. 2020, MNRAS, 492, 1594

Suri, S., Sánchez-Monge, Á., Schilke, P., et al. 2019, A\&A, 623, A142

Taylor, J. H., \& Cordes, J. M. 1993, ApJ, 411, 674

Urquhart, J. S., Figura, C., Cross, J. R., et al. 2021, MNRAS, 500, 3050

Virtanen, P., Gommers, R., Oliphant, T. E., et al. 2020, Nat. Methods, 17, 261

Wang, K., Testi, L., Ginsburg, A., et al. 2015, MNRAS, 450, 4043

Wang, K., Testi, L., Burkert, A., et al. 2016, ApJS, 226, 9

Wilson, T. L., Rohlfs, K., \& Hüttemeister, S. 2013, Tools of Radio Astronomy

Zhang, M., Kainulainen, J., Mattern, M., Fang, M., \& Henning, T. 2019, A\&A, 622, A52

Zucker, C., Battersby, C., \& Goodman, A. 2015, ApJ, 815, 23

Zucker, C., Battersby, C., \& Goodman, A. 2018, ApJ, 864, 153

Zucker, C., Smith, R., \& Goodman, A. 2019, ApJ, 887, 186 


\section{Appendix A: A glimpse into the outer Galaxy environment}

In addition to the decrements of molecular gas surface density and the molecular cloud mass across the Galactic disc (see Section 3), several studies have shown that many other parameters display gradients with respect to the Galactocentric radius, $R_{\mathrm{Gal}}$, making the outer Galaxy an environment profoundly different compared to the inner Milky Way. For example, Giannetti et al. (2017) measured a gas-to-dust, $\gamma$, gradient $\log (\gamma)=0.087 R_{\mathrm{Gal}}+1.44$. Such a gradient would imply a large increment of the gas-to-dust ratio from the inner to the outer Galaxy with, for example, $\gamma\left(R_{\mathrm{Gal}}=5 \mathrm{kpc}\right) \sim 75$ and $\gamma\left(R_{\mathrm{Gal}}=15 \mathrm{kpc}\right) \sim 556$. At the same time, they inferred the variation of gas metallicity, $Z$, with respect to $R_{\mathrm{Gal}}: \log (Z)=$ $-0.056 R_{\mathrm{Gal}}-1.176$. Considering this, the metallicity at the Sun location $\left(R_{\mathrm{Gal}}=8.1 \mathrm{kpc}\right)$ is $\sim 2.5$ higher than the metallicity at $R_{\mathrm{Gal}}=15 \mathrm{kpc}$. Various works (see Kalberla \& Kerp 2009 , and references therein) have reported decrements of the HI surface density $\Sigma_{\mathrm{HI}}=30 \exp \left(\left(R_{\mathrm{Gal}}-R_{0}\right) / 3.75\right) \mathrm{M}_{\odot} \mathrm{pc}^{-2}$. This quantity, however, appears to saturate at $\Sigma_{\mathrm{HI}} \sim 10 \mathrm{M}_{\odot} \mathrm{pc}^{-2}$ up to $R_{\mathrm{Gal}}=15 \mathrm{kpc}$. A reduced radiation field has also been measured in the outer Galaxy. For example, as the $\gamma$-ray emissivity appears to be directly correlated with the HI column density, Bloemen et al. (1984) found that $\gamma$-ray emissivity decreases by $15 \%$ for $R_{\mathrm{Gal}}>R_{0}$ with only $25 \%$ of the $\gamma$-ray intensity coming from $14<R_{\mathrm{Gal}}<17 \mathrm{kpc}$.

\section{Appendix B: Data and methods of analysis}

\section{B.1. OGHReS science demonstration data}

OGHReS is a large programme that is mapping the ${ }^{12} \mathrm{CO}(2-1)$, ${ }^{13} \mathrm{CO}(2-1)$, and $\mathrm{C}^{18} \mathrm{O}(2-1)$ emission across $180 \leq l \leq 280 \mathrm{deg}$ using the PI230 and nFLASH230 receivers mounted on the Atacama Pathfinder EXperiment (APEX, Güsten et al. 2006). The latitude coverage varies and follows the Galactic warp, covering $-2.0<b<-1.0 \mathrm{deg}$ in the range of $280>\ell>225 \mathrm{deg}$ then increasing in latitude up to $\ell=205 \mathrm{deg}$ from where it covers $-0.5<b<0.5 \mathrm{deg}$ down to $\ell=180 \mathrm{deg}$. Data present an angular resolution HPBW $=27^{\prime \prime}$, a spectral resolution of $0.25 \mathrm{~km} \mathrm{~s}^{-1}$, and a median $\sigma_{\text {RMS }}=\mathbf{0 . 2 3} \pm \mathbf{0 . 0 8} \mathrm{K}\left(\mathrm{T}_{\mathrm{mb}}\right)$. Here, we use data from the science demonstration phase of the survey (OGHReS SD1), covering $250 \leq l \leq 280 \mathrm{deg}$ (see König et al. in prep. for more details).

\section{B.2. Inner Galaxy large-scale filament data}

In the paper, we compare the location and properties of the OGLSFs considering the inner Galaxy LSFs (IGLSFs), which were collected and homogeneously reanalysed by Zucker et al. (2018), consisting of 45 objects. As in Zucker et al. (2018), here we adopted the same terminology to separate the IGLSFs as those structures that have been identified using different methods and that present slightly different properties (see Fig. 1 in Zucker et al. 2018 for further details). Those LSFs are named giant molecular filaments (GMFs, Ragan et al. 2014; Abreu-Vicente et al. 2016), large-scale Herschel filaments (Wang et al. 2015), Milky Way 'bones' (Zucker et al. 2015), and minimum spanning tree (MST) filaments (Wang et al. 2016). To this sample, we added the SEDIGISM (Schuller et al. 2021) filaments assembled by Mattern et al. (2018b), starting from dustcontinuum filaments originally extracted from ATLASGAL data (Schuller et al. 2009; Li et al. 2016). To be consistent with our sample that of Zucker et al. (2018), we considered only the fully correlated, single component filaments in the SEDIGISM catalogue that have a length $>10 \mathrm{pc}$, for a total of 30 objects. The final sample of IGLSFs consists of 75 objects. For consistency, we only considered objects whose coherency has been verified in emission. Therefore, here, we do not use larger filament samples, such as the Hi-GAL catalogue presented in Schisano et al. (2020). For comparison, we also show the property measurements performed by Zhang et al. (2019) by using the same LSFs considered by Zucker et al. (2018) (e.g. excluding the ATLASGAL filaments from Zhang et al. 2019, which have been homogeneously and fully reanalysed by Mattern et al. 2018b).

\section{B.3. Outer Galaxy large-scale filament identification and property calculation}

To search for LSFs within OGHReS data, we used a procedure consisting of several steps. Following Zucker et al. (2018), we looked for coherent structures with a clear filamentary morphology (or an aspect ratio, $A R \gtrsim 10$ ) and length $\gtrsim 10$ pc. In this work, we are only interested in 'outer Galaxy' LSFs (or OGLSFs), therefore we generally only considered the emission with $V_{\mathrm{LSR}}>40 \mathrm{~km} \mathrm{~s}^{-1}$.

Firstly, we masked the data using a 'dilate mask' technique (Rosolowsky \& Leroy 2006), where we retained connected regions in the datacubes, whose pixels show a peak $\mathrm{S} / \mathrm{N}>$ 2 , but also contain a region with $\mathrm{S} / \mathrm{N}>4$. The $\mathrm{S} / \mathrm{N}$ was calculated line-of-sight-wise and the noise map was obtained considering the standard deviation of the first and last ten line-free channels in the datacubes (which constitutes $\sim 2 \%$ of the total channels in the datacubes). A dendrogram analysis ${ }^{1}$ was performed in order to label the isolated regions in the masked data and to calculate their properties. In particular, the dendrogram produces three-dimensional (position-position-velocity) masks of the catalogued hierarchical structures, where each voxel that belongs to a given structure is labelled with the structure ID. The bidimensional projections of the structures on the plane of the sky were generated by flattening those 3D masks across the velocity dimension. We then used these bi-dimensional masks to measure the structures length. In order to compensate for low S/N pixels that were masked in the previous step, we homogenised the bi-dimensional mask using SCIPY BINARY_FILL_HOLES task ${ }^{2}$. Applying the medial axis analysis implemented in the FilFinder2D package ${ }^{3}$ (Koch \& Rosolowsky 2015), we found the longest path across the skeleton of the structures, which constitute their spine. The length of the spine is equivalent to the length of the structure. The medial axis analysis works by labelling each pixel in the bi-dimensional projected mask with a number based on the distance (in pixels) to the structure boundary. Pixels within the mask with the largest distance from the boundary form the skeleton of the structure. The structure width was calculated as $2 \times$ the median of the distance (in pixels) from the spine to the outer edge of the structure bi-dimensional mask (following Duarte-Cabral et al. 2021). For consistency, we also calculated the width as a median of the lengths of the spine 'perpendicular cuts' implemented in the RadFil package ${ }^{4}$. We found that our

\footnotetext{
1 Using ASTRODENDRO, https://dendrograms.readthedocs. io/en/stable/

2 https://docs.scipy.org/doc/scipy-0.14.0/reference/ generated/scipy.ndimage.morphology . binary_fill_holes. html

3 https://fil-finder.readthedocs.io/en/latest/

4 https://github.com/catherinezucker/radfil
} 
method tends to slightly underestimate the width of the structures compared to RadFil; however, the two measurements are largely comparable (see Table C.1 in Appendix C). The kinematic heliocentric distance to the structures was measured using the Brand \& Blitz (1993) Galaxy rotation curve model using $V_{0}=240 \mathrm{~km} \mathrm{~s}^{-1}$ from Reid et al. (2014), which is consistent (within the uncertainties) with the updated value from Reid et al (2019), $V_{0}=236 \pm 7 \mathrm{~km} \mathrm{~s}^{-1}$. Also, we assumed $R_{0}=8.15 \mathrm{kpc}$ (Reid et al. 2019).

\section{B.4. Mass estimates}

The mass of a filament or generally of a molecular gas structure can be derived in a variety of ways, each of them presenting its own biases and approximations. For example, masses derived from dust continuum, such as in the case of the inner Galaxy filaments of Zucker et al. (2018), might be affected by foreground and background subtraction uncertainties. Mattern et al. (2018a) and Zhang et al. (2019) used the ${ }^{13} \mathrm{CO}$ to derive the molecular gas mass. The ${ }^{13} \mathrm{CO}$ transition is sensitive to a denser medium than the ${ }^{12} \mathrm{CO}$ transition (implied in our study), and it is less affected by optical depth effects. In turn, ${ }^{13} \mathrm{CO}$ suffers from more severe lower beam filling factor effects than ${ }^{12} \mathrm{CO}$. In essence, all methods aim to measure the $\mathrm{H}_{2}$ column density, $N\left(\mathrm{H}_{2}\right)$, from which the molecular gas mass is calculated as follows:

$M=\mu_{\mathrm{H}_{2}} m_{\mathrm{p}} \sum_{l b v} N\left(\mathrm{H}_{2}\right) \Delta x \Delta y$,

where the mean molecular weight of the molecular hydrogen is $\mu_{\mathrm{H}_{2}}=2.8, m_{\mathrm{p}}$ is the proton mass (i.e. the mass of the hydrogen atom), and $\Delta x$ and $\Delta y$ represent the pixel size. Benedettini et al. (2021) used three methods to derive the mass of the clouds in the outer Galaxy (for $220<l<240 \mathrm{deg}$ ) for the Forgotten Quadrant Survey (FQS), finding that they all give very similar mass estimates. They calculated $N\left(\mathrm{H}_{2}\right)$ from the ${ }^{12} \mathrm{CO}(1-0)$ emission, from the ${ }^{13} \mathrm{CO}(1-0)$ column density, and from the far-infrared dust emission. This study is particularly relevant for our purposes since the FQS observed the molecular medium of the outer Milky Way in a Galactic region that will also be covered by the full OGHReS data once the survey is completed.

For our OGLSFs, we chose to calculate the molecular gas mass from ${ }^{12} \mathrm{CO}(2-1)$ intensity assuming a ${ }^{12} \mathrm{CO}$-to- $\mathrm{H}_{2}$ conversion factor $X_{\mathrm{CO}}$. This method provides the $\mathrm{H}_{2}$ column density as follows:

$N\left(\mathrm{H}_{2}\right)=X_{\mathrm{CO}(1-0)} I_{\mathrm{CO}(2-1)} R_{21}^{-1}$,

where $I_{\mathrm{CO}(2-1)}$ is the integrated intensity of the ${ }^{12} \mathrm{CO}(2-1)$ emission. We assumed $X_{\mathrm{CO}(1-0)}=2.3 \times 10^{20} \mathrm{~cm}^{-2}\left(\mathrm{~K} \mathrm{~km} \mathrm{~s}^{-1}\right)^{-1}$ inferred by Brand \& Wouterloot (1995) (see also Heyer et al. 2001; Bolatto et al. 2013; König et al. 2021) who showed that in the outer Galaxy, the CO-to- $\mathrm{H}_{2}$ conversion factor, $X_{\mathrm{CO}(1-0)}$, is within the uncertainties consistent with the inner Galaxy value. As $X_{\mathrm{CO}(1-0)}$ was calculated for the ${ }^{12} \mathrm{CO}(1-0)$ line, we used a ration between the ${ }^{12} \mathrm{CO}(2-1)$ to the ${ }^{12} \mathrm{CO}(1-0)$ intensity, $R_{21}=$ 0.7 (e.g. Sandstrom et al. 2013; Nishimura et al. 2015).

Modern studies, based on high resolution surveys, have shown that $X_{\mathrm{CO}}$ is not constant, by it varies pixel-by-pixel (e.g Pitts \& Barnes 2021), and it diverges from the assumed value especially at low integrated intensity. Therefore, here we calculate $N\left(\mathrm{H}_{2}\right)$ from the ${ }^{13} \mathrm{CO}$ column density. Unfortunately, for our sources, the ${ }^{13} \mathrm{CO}$ emission was only detected across the brightest regions within the filaments and not for every filament. Therefore, ${ }^{13} \mathrm{CO}$ emission cannot be used to infer the whole mass of the OGLSFs, identified through the more extended and brighter ${ }^{12} \mathrm{CO}$ emission, but only to test the reliability of our masses estimated through the ${ }^{12} \mathrm{CO}$ emission. To do so, we first isolated the high $\mathrm{S} / \mathrm{N}{ }^{13} \mathrm{CO}$ emission using a dilate masking technique (see Section B.3) considering $\mathrm{S} / \mathrm{N}=2,3$ for the low and high masking thresholds, respectively. We then assumed that the molecular gas can be described as a system in local thermodymanic equilibrium (LTE) and that the ${ }^{12} \mathrm{CO}(2-1)$ is optically thick. According to the formalism by Wilson et al. (2013), the excitation temperature, $T_{\text {ex }}$, at every voxel $(l, b, v)$ can be derived as follows:

$T_{\mathrm{ex}}[K]=\frac{11.06}{\ln \left[1+11.06 /\left(T_{\mathrm{mb}}\left({ }^{12} \mathrm{CO}\right)+0.194\right)\right]}$,

where $T_{\mathrm{mb}}\left({ }^{12} \mathrm{CO}\right.$ is the main-beam brightness temperature of the ${ }^{12} \mathrm{CO}$ emission at a given $(l, b, v)$ voxel. This equation is derived from equation 15.29 from Wilson et al. (2013) considering a cosmic background temperature $T_{\mathrm{bg}}=2.725 \mathrm{~K}$ and $v_{12} \mathrm{CO}(2-1)=230.538 \mathrm{GHz}$.

The optical depth of the ${ }^{13} \mathrm{CO}(2-1)$ is calculated at every $(l, b, v)$ voxel, solving equation 15.29 from Wilson et al. (2013) for $\tau$ :

$\tau_{13}=-\ln \left[1-\frac{T_{\mathrm{mb}}\left({ }^{13} \mathrm{CO}\right) / 10.58}{\left(\exp \left(10.58 / T_{\mathrm{ex}}\right)-1\right)^{-1}-0.02}\right]$,

where $T_{\mathrm{mb}}\left({ }^{13} \mathrm{CO}\right.$ is the main-beam brightness temperature of the ${ }^{13} \mathrm{CO}$ emission at a given $(l, b, v)$ voxel.

We derived the total ${ }^{13} \mathrm{CO}$ column density following Wilson et al. (2013), equation 15.37:

$N\left({ }^{13} \mathrm{CO}\right)\left[\mathrm{cm}^{-2}\right]=1.5 \times 10^{4} \int \frac{T_{\mathrm{ex}} \exp \left(5.3 / T_{\mathrm{ex}}\right)}{1-\exp \left(-10.6 / T_{\mathrm{ex}}\right)} \tau_{13} \mathrm{~d} v$,

where the velocity, $v$, is in $\mathrm{km} \mathrm{s}^{-1}$, and the integration runs across the full spectral extent of the structure. The column density of the molecular gas follows as:

$N\left(\mathrm{H}_{2}\right)\left[\mathrm{cm}^{-2}\right]=\frac{\left[\mathrm{H}_{2}\right]}{\left[{ }^{13} \mathrm{CO}\right]} N\left({ }^{13} \mathrm{CO}\right)$,

where $\left[\mathrm{H}_{2}\right] /\left[{ }^{13} \mathrm{CO}\right]=7.1 \times 10^{5}$ is the isotopic abundance ratio of the ${ }^{13} \mathrm{CO}$ isotopologue relative to $\mathrm{H}_{2}$ (Frerking et al. 1982).

A further alternative is to infer $N\left(\mathrm{H}_{2}\right)$ from the ${ }^{13} \mathrm{CO}(2-1)$ integrated emission directly, by assuming a constant $X_{{ }^{13} \mathrm{CO}(2-1)}$ factor. Such a $X$-factor for the ${ }^{13} \mathrm{CO}(2-1)$ line has been calculated from SEDIGISM survey data (Schuller et al. 2017). On average, the $X_{{ }^{13} \mathrm{CO}(2-1)}$ they measured appears roughly constant with the intensity (even if a large scatter is observed). Schuller et al. (2017) calculated the $X$-factor in two ways, comparing Hi-GAL dust continuum emission and ${ }^{13} \mathrm{CO}(2-1)$ emission, and via a radiative transfer solution involving Three-mm Ultimate Mopra Milky Way Survey (ThrUMMS) data (Barnes et al. 2015). Both methods infer an average $X$-factor consistent with $X_{{ }^{13} \mathrm{CO}(2-1)}=1 \times 10^{21} \mathrm{~cm}^{-2}\left(\mathrm{~K} \mathrm{~km} \mathrm{~s}^{-1}\right)^{-1}$. Assuming that $X_{{ }^{13} \mathrm{CO}(2-1)}$ does not present a significant variation with respect to the Galactocentric radius (such as $X_{\mathrm{CO}(1-0)}$ ), we can calculate $N\left(\mathrm{H}_{2}\right)$ as follows:

$N\left(\mathrm{H}_{2}\right)\left[\mathrm{cm}^{-2}\right]=X_{13} \mathrm{CO}(2-1){ }^{I_{13}} \mathrm{CO}(2-1)$.

We named the mass estimates as $M_{X\left({ }^{12} \mathrm{CO}\right)}, M_{N\left({ }^{13} \mathrm{CO}\right)}$, and $M_{X\left({ }^{13} \mathrm{CO}\right)}$, considering $N\left(\mathrm{H}_{2}\right)$ inferred from equations B.2, B.6, and B.7, respectively. To perform this test, $M_{X\left({ }^{12} \mathrm{CO}\right)}$ was calculated across the same area defined by the ${ }^{13} \mathrm{CO}(2-1)$ emission mask for each filament in our sample. 
Table B.1. Ratio of the masses of the clumps within the OGLSFs derived using various methods.

\begin{tabular}{lccc}
\hline \hline Mass ratio & Median & $25^{\text {th }}$ percentile & $75^{\text {th }}$ percentile \\
\hline$M_{X\left({ }^{12} \mathrm{CO}\right)} / M_{N\left({ }^{13} \mathrm{CO}\right)}$ & 1.08 & 0.83 & 1.49 \\
$M_{X\left({ }^{12} \mathrm{CO}\right)} / M_{X\left({ }^{13} \mathrm{CO}\right)}$ & 1.00 & 0.88 & 1.14 \\
\hline
\end{tabular}

Notes. See description in Section B.4.

Medians and percentile values for the mass ratio distributions calculated through the three methods discussed here are summarised in Table B.1. Generally, the masses estimated from the ${ }^{13} \mathrm{CO}$ emission and column density largely agree with the masses inferred from the ${ }^{12} \mathrm{CO}$ emission assuming a constant $X$-factor. The most significant variations are observed for filaments where the ${ }^{13} \mathrm{CO}$ emission is barely detected. Given this, we expect the masses derived for the whole OGLSFs through the ${ }^{12} \mathrm{CO}$ emission to be a reliable estimation of the actual molecular gas mass of the structures.

However, looking at Fig. 3, it appears that different mass calculation methods give different estimates for the filaments in the inner Galaxy. LSF masses from Zhang et al. (2019) appear to be a factor of a few larger than the masses measured by Zucker et al. (2018) (see Fig. 3). On average, however, the lengths are more comparable. This results in larger linear masses than the ones inferred by Zucker et al. (2018) (further increasing the difference between inner and outer Galaxy LSF linear masses). Zucker et al. (2018) used HI-GAL data to trace the filament boundaries (and infer their masses from $N\left(\mathrm{H}_{2}\right)$ inferred from dust continuum), in an attempt to maintain the structure shape and appearance compared to their original studies. In turn, Zhang et al. (2019) measured the LSF properties from ${ }^{13} \mathrm{CO}(1-0)$ data (including their mass through $N\left({ }^{13} \mathrm{CO}\right)$ ), assuming a constant $V$-band extinction, $A_{\mathrm{v}}=3$, to define the boundary of each structure in their sample. In this aspect, Zucker et al. (2018) consider the denser part of the filaments, resulting in lower masses and linear masses. Therefore, discrepancies between the measurements of Zucker et al. (2018) and Zhang et al. (2019) are possibly due to the different methods used to define the LSFs, rather than inconsistencies between dust and ${ }^{13} \mathrm{CO}$ emission to calculate the structure masses. Indeed, for the GMFs, for which both works used the structure masks from the original studies, mass distributions appear more comparable. 


\section{Appendix C: Catalogue of properties and integrated intensity map atlas of the outer Galaxy large-scale filaments}

Here, we provide in Table C.1 the catalogue of the OGLSF properties discussed in the paper. Together we show the remaining OGLSF integrated intensity maps (Fig. C.1-C.5). Sub-cubes containing the ${ }^{12} \mathrm{CO}(2-1)$ emission from the 37 OGLSFs can be obtained from CDS.

Table C.1. Catalogue of the OGLSFs identified within the OGHReS SD1 field

\begin{tabular}{|c|c|c|c|c|c|c|c|c|c|c|c|c|c|}
\hline Name & $\begin{array}{l}l \\
\circ\end{array}$ & $\begin{array}{l}b \\
\circ\end{array}$ & $\begin{array}{c}V_{\mathrm{LSR}} \\
\mathrm{km} \mathrm{s}^{-1}\end{array}$ & $\begin{array}{c}D \\
\mathrm{kpc}\end{array}$ & $\begin{array}{l}x_{\text {Gal }} \\
\mathrm{kpc}\end{array}$ & $\begin{array}{l}y_{\text {Gal }} \\
\mathrm{kpc}\end{array}$ & $\begin{array}{c}\sigma_{\mathrm{v}} \\
\mathrm{km} \mathrm{s}^{-1}\end{array}$ & $\begin{array}{c}V_{5 \text { th }} / V_{95 \text { th }} \\
\mathrm{km} \mathrm{s}^{-1}\end{array}$ & $\begin{array}{c}\log \left(M_{\mathrm{CO}}\right) \\
M_{\odot}\end{array}$ & $\begin{array}{l}\text { length } \\
\mathrm{pc}\end{array}$ & $A R_{\mathrm{MA}}$ & $A R_{\text {RadFil }}$ & $\begin{array}{l}\Delta V_{\text {sp.arm }} \\
\mathrm{km} \mathrm{s}^{-1}\end{array}$ \\
\hline OGLSF251.38-1.76 & 251.38 & -1.76 & 49.2 & 4.34 & -4.11 & 9.49 & 0.6 & $48 / 50$ & 3.00 & 22.5 & 13.4 & 11.3 & 27.9 \\
\hline OGLSF254.17-1.07 & 254.17 & -1.07 & 77.3 & 7.15 & -6.88 & 10.05 & 1.0 & $75 / 79$ & 2.79 & 24.3 & 15.7 & 13.9 & 2.5 \\
\hline OGLSF255.47-1.07 & 255.47 & -1.07 & 80.2 & 7.53 & -7.29 & 9.99 & 0.7 & $78 / 81$ & 2.60 & 22.6 & 13.9 & 10.7 & 0.8 \\
\hline OGLSF256.69-1.62 & 256.69 & -1.62 & 42.8 & 4.16 & -4.04 & 9.06 & 0.8 & $41 / 44$ & 2.82 & 22.0 & 17.2 & 16.0 & 39.2 \\
\hline OGLSF257.86-1.94 & 257.86 & -1.94 & 57.8 & 5.57 & -5.44 & 9.27 & 0.5 & $57 / 58$ & 2.53 & 18.1 & 11.2 & 10.6 & 25.3 \\
\hline OGLSF258.13-1.58 & 258.13 & -1.58 & 41.5 & 4.16 & -4.07 & 8.96 & 1.1 & $39 / 43$ & 3.26 & 26.8 & 12.3 & 11.1 & 41.8 \\
\hline OGLSF259.12-1.85 & 259.12 & -1.85 & 54.6 & 5.39 & -5.30 & 9.12 & 1.1 & $52 / 56$ & 3.58 & 28.0 & 12.0 & 9.7 & 29.6 \\
\hline OGLSF259.82-1.38 & 259.82 & -1.38 & 61.5 & 6.07 & -5.98 & 9.17 & 1.5 & $58 / 63$ & 3.48 & 55.9 & 26.3 & 26.0 & 23.4 \\
\hline OGLSF260.02-1.25 & 260.02 & -1.25 & 63.0 & 6.23 & -6.14 & 9.18 & 1.9 & $59 / 65$ & 3.51 & 45.0 & 14.6 & 10.7 & 22.0 \\
\hline OGLSF260.31-1.26 & 260.31 & -1.26 & 62.9 & 6.25 & -6.16 & 9.15 & 0.7 & $61 / 64$ & 3.14 & 25.1 & 10.4 & 10.0 & 22.4 \\
\hline OGLSF261.31-1.08 & 261.31 & -1.08 & 66.0 & 6.64 & -6.56 & 9.10 & 0.9 & $64 / 67$ & 3.57 & 44.8 & 11.6 & 10.7 & 20.1 \\
\hline OGLSF261.34-1.17 & 261.34 & -1.17 & 56.7 & 5.79 & -5.73 & 8.97 & 1.2 & $54 / 58$ & 2.48 & 13.2 & 10.5 & 10.4 & 29.4 \\
\hline OGLSF263.42-1.43 & 263.42 & -1.43 & 55.5 & 5.91 & -5.87 & 8.78 & 0.4 & $54 / 56$ & 2.12 & 12.9 & 10.1 & 10.8 & 32.3 \\
\hline OGLSF265.59-1.48 & 265.59 & -1.48 & 63.7 & 6.90 & -6.88 & 8.63 & 0.8 & $62 / 65$ & 3.64 & 66.2 & 22.1 & 14.7 & 25.8 \\
\hline OGLSF265.78-1.20 & 265.78 & -1.20 & 62.5 & 6.81 & -6.79 & 8.60 & 1.5 & $58 / 64$ & 3.67 & 51.8 & 15.4 & 12.4 & 27.2 \\
\hline OGLSF266.69-1.21 & 266.69 & -1.21 & 42.1 & 5.11 & -5.11 & 8.40 & 0.8 & $40 / 43$ & 2.77 & 17.6 & 12.6 & 12.3 & 48.3 \\
\hline OGLSF269.04-1.25 & 269.04 & -1.25 & 61.9 & 7.18 & -7.18 & 8.22 & 1.6 & $59 / 65$ & 3.64 & 45.3 & 13.0 & 11.7 & 30.2 \\
\hline OGLSF269.31-1.72 & 269.31 & -1.72 & 82.3 & 9.19 & -9.19 & 8.21 & 1.3 & $80 / 84$ & 3.91 & 57.5 & 15.6 & 13.7 & 10.0 \\
\hline OGLSF269.38-1.62 & 269.38 & -1.62 & 80.0 & 8.97 & -8.97 & 8.20 & 0.6 & $79 / 81$ & 2.65 & 18.7 & 9.6 & 11.3 & 12.3 \\
\hline OGLSF269.40-1.43 & 269.40 & -1.43 & 59.3 & 7.00 & -7.00 & 8.17 & 2.0 & $55 / 62$ & 3.74 & 43.4 & 11.9 & 10.8 & 33.0 \\
\hline OGLSF269.92-1.38 & 269.92 & -1.38 & 54.6 & 6.65 & -6.65 & 8.11 & 0.6 & $53 / 55$ & 2.45 & 14.5 & 10.1 & 10.5 & 38.1 \\
\hline OGLSF270.23-1.27 & 270.23 & -1.27 & 52.8 & 6.53 & -6.53 & 8.07 & 1.1 & $50 / 54$ & 3.03 & 24.1 & 10.0 & 8.6 & 40.1 \\
\hline OGLSF270.26-1.50 & 270.26 & -1.50 & 54.7 & 6.71 & -6.71 & 8.07 & 3.7 & $48 / 59$ & 4.81 & 111.9 & 12.8 & 10.3 & 38.2 \\
\hline OGLSF270.97-1.85 & 270.97 & -1.85 & 44.8 & 5.93 & -5.93 & 8.00 & 0.9 & $43 / 46$ & 3.59 & 33.9 & 11.6 & 9.1 & 48.6 \\
\hline OGLSF272.03-1.56 & 272.03 & -1.56 & 73.7 & 8.72 & -8.72 & 7.79 & 0.7 & $72 / 74$ & 2.43 & 17.8 & 14.8 & 14.5 & 20.4 \\
\hline OGLSF272.81-1.43 & 272.81 & -1.43 & 39.9 & 5.78 & -5.77 & 7.82 & 0.8 & $38 / 41$ & 2.94 & 24.9 & 12.3 & 11.5 & 54.7 \\
\hline OGLSF273.05-1.63 & 273.05 & -1.63 & 55.8 & 7.22 & -7.21 & 7.72 & 0.7 & $54 / 57$ & 2.54 & 15.1 & 9.6 & 8.6 & 38.9 \\
\hline OGLSF274.07-1.20 & 274.07 & -1.20 & 38.5 & 5.86 & -5.84 & 7.68 & 2.4 & $34 / 42$ & 4.82 & 143.4 & 20.8 & 17.0 & 56.8 \\
\hline OGLSF274.27-1.45 & 274.27 & -1.45 & 77.3 & 9.43 & -9.40 & 7.40 & 1.3 & $75 / 79$ & 3.67 & 36.6 & 12.6 & 10.4 & 18.1 \\
\hline OGLSF274.94-1.32 & 274.94 & -1.32 & 87.1 & 10.56 & -10.52 & 7.19 & 0.7 & $86 / 88$ & 3.00 & 29.7 & 10.3 & 10.8 & 8.7 \\
\hline OGLSF275.32-1.69 & 275.32 & -1.69 & 56.4 & 7.63 & -7.60 & 7.39 & 1.1 & $54 / 58$ & 3.87 & 57.3 & 11.0 & 9.5 & 39.7 \\
\hline OGLSF275.37-1.09 & 275.37 & -1.09 & 70.7 & 8.96 & -8.92 & 7.26 & 1.6 & $68 / 73$ & 3.66 & 40.4 & 12.9 & 11.9 & 25.4 \\
\hline OGLSF275.39-1.11 & 275.39 & -1.11 & 61.9 & 8.14 & -8.10 & 7.33 & 0.9 & $60 / 63$ & 3.15 & 28.8 & 10.1 & 8.7 & 34.2 \\
\hline OGLSF275.95-1.77 & 275.95 & -1.77 & 56.6 & 7.75 & -7.71 & 7.30 & 3.7 & $51 / 63$ & 4.46 & 80.4 & 12.5 & 9.7 & 39.8 \\
\hline OGLSF278.85-1.33 & 278.85 & -1.33 & 45.8 & 7.31 & -7.22 & 6.97 & 1.2 & $43 / 48$ & 4.10 & 64.1 & 14.9 & 11.0 & 52.0 \\
\hline OGLSF279.42-1.19 & 279.42 & -1.19 & 39.9 & 6.90 & -6.80 & 6.97 & 0.9 & $38 / 41$ & 3.41 & 32.8 & 12.2 & 12.5 & 58.1 \\
\hline OGLSF279.64-1.51 & 279.64 & -1.51 & 16.8 & 4.85 & -4.78 & 7.29 & 0.6 & $16 / 18$ & 2.63 & 18.1 & 13.6 & 12.8 & 81.4 \\
\hline
\end{tabular}

Notes. From left to right: name of the source (Name); Galactic longitude $(l)$; Galactic latitude $(b)$; LSR velocity $\left(V_{\text {LSR }}\right)$; heliocentric distance $(D)$; Galactocentric $x$ coordinate $\left(x_{\mathrm{Gal}}\right)$; Galactocentric $y$ coordinate $\left(y_{\mathrm{Gal}}\right)$; velocity dispersion $\left(\sigma_{\mathrm{v}}\right)$; fifth and 95th percentile of the velocity distribution span by a filament $\left(V_{5 \text { th }}\right.$ and $V_{95 \text { th }}$, respectively); logarithmic mass from the CO luminosity $\left(M_{\mathrm{CO}}\right)$; filament length (length); aspect ratio with the width inferred from the medial axis analysis $\left(A R_{\mathrm{MA}}\right)$; aspect ratio with the width inferred from RadFil $\left(A R_{\text {RadFil }}\right)$; and velocity offset with respect to Perseus spiral arm considering Taylor \& Cordes (1993) models $\left(\Delta V_{\text {sp.arm }}\right)$. 

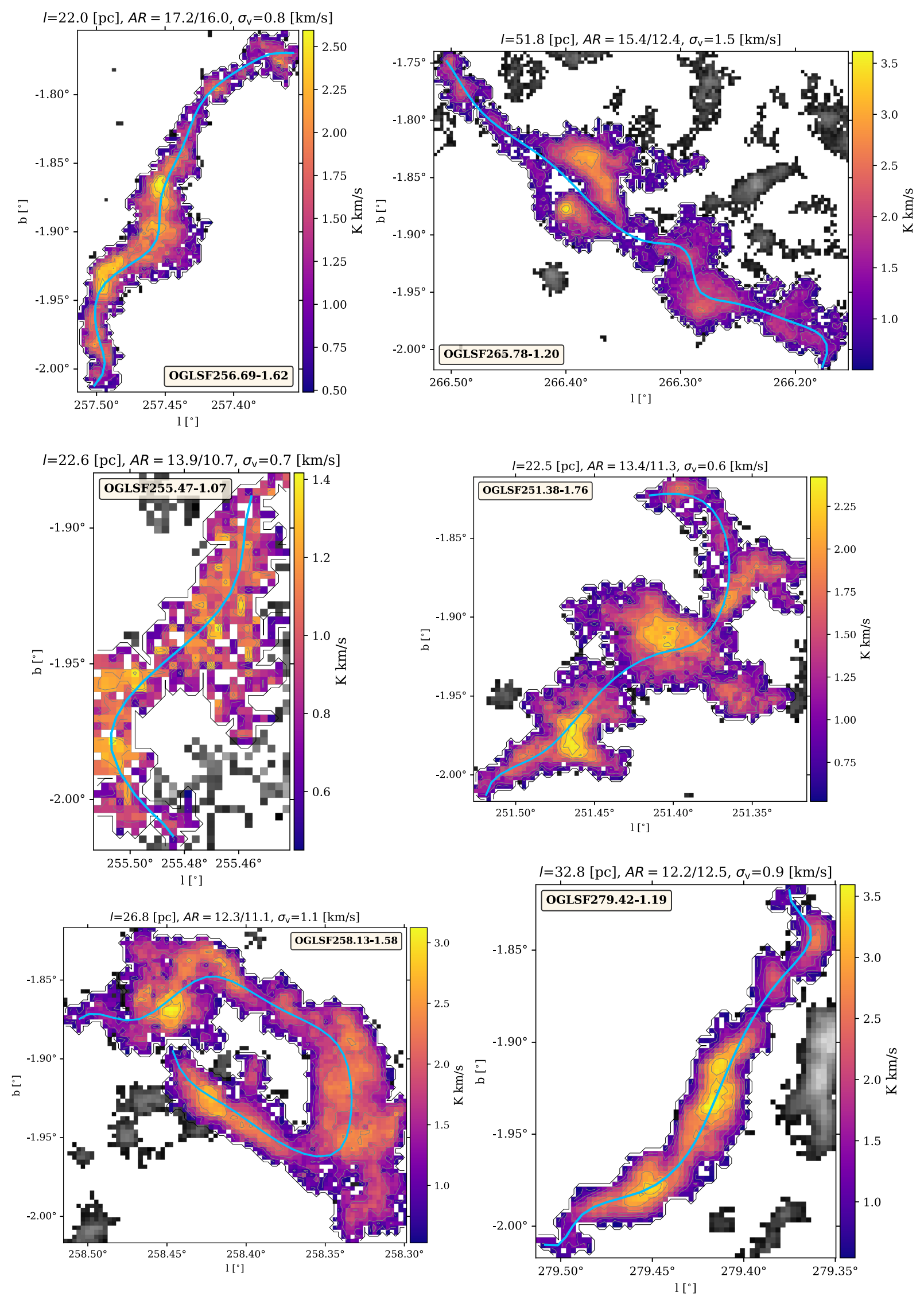

Fig. C.1. Integrated intensity maps of the ${ }^{12} \mathrm{CO}(2-1)$ emission from OGLSFs. Symbols and conventions are the same as in Fig. 1. 

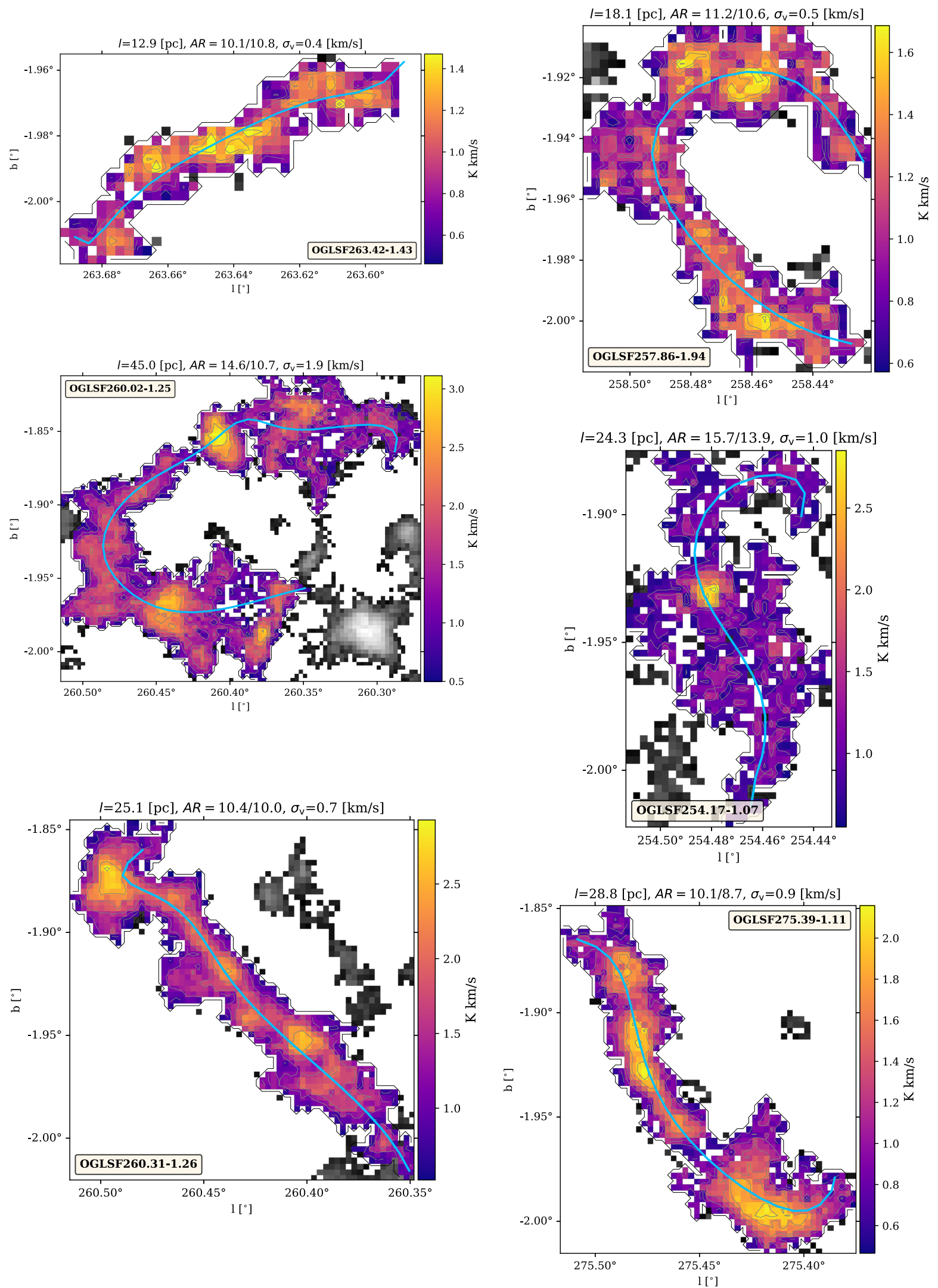

Fig. C.2. Integrated intensity maps of the ${ }^{12} \mathrm{CO}(2-1)$ emission from OGLSFs. Symbols and conventions are the same as in Fig. 1 . 

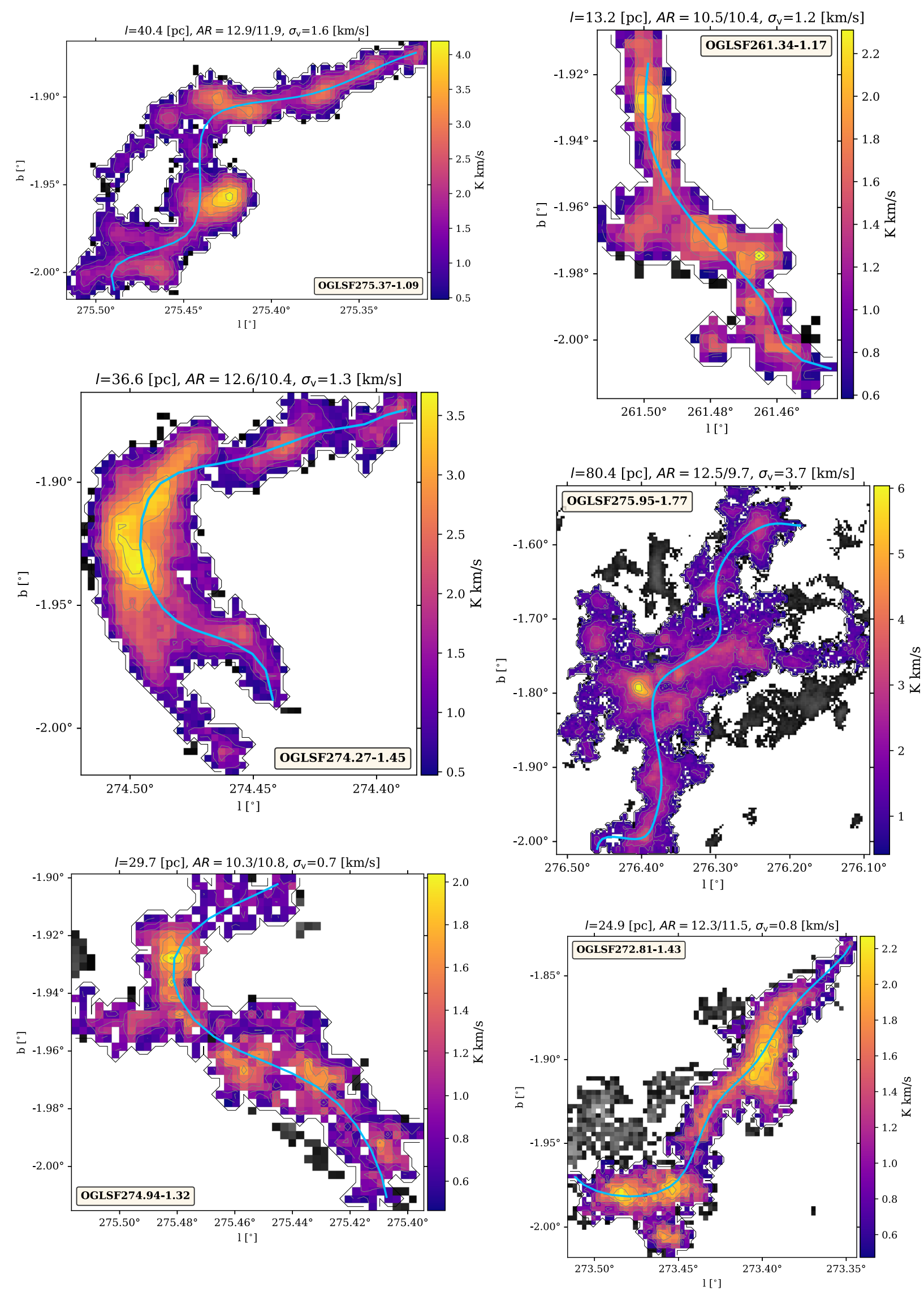

Fig. C.3. Integrated intensity maps of the ${ }^{12} \mathrm{CO}(2-1)$ emission from OGLSFs. Symbols and conventions are the same as in Fig. 1. 
D. Colombo et al.: OGHReS: Outer Galaxy large-scale filaments

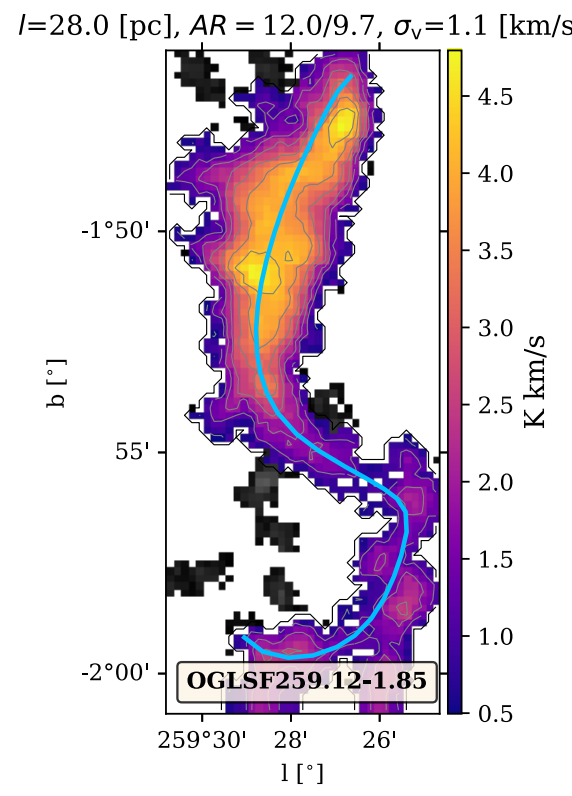

$I=45.3[\mathrm{pc}], A R=13.0 / 11.7, \sigma_{\mathrm{v}}=1.6[\mathrm{~km} / \mathrm{s}]$
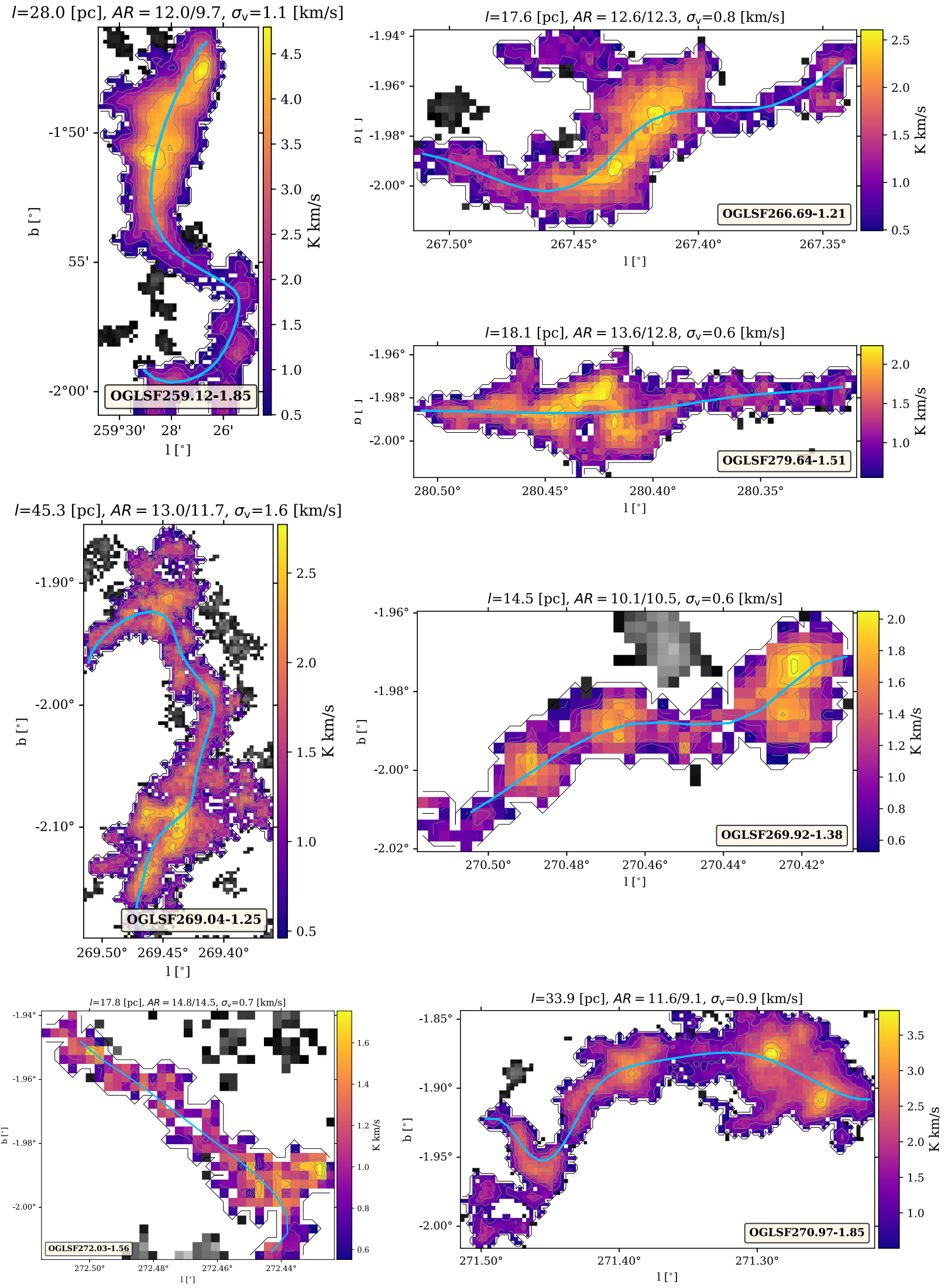

Fig. C.4. Integrated intensity maps of the ${ }^{12} \mathrm{CO}(2-1)$ emission from OGLSFs. Symbols and conventions are the same as in Fig. 1. 


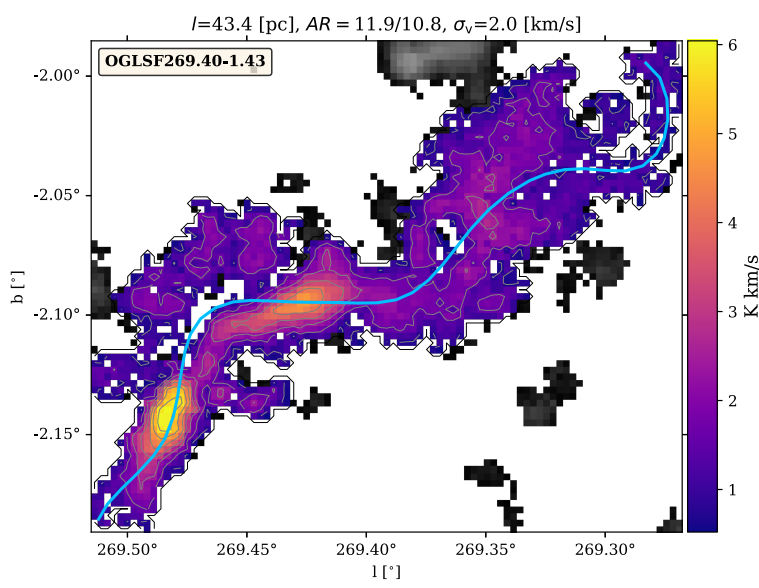

$I=15.1[\mathrm{pc}], A R=9.6 / 8.6, \sigma_{\mathrm{v}}=0.7[\mathrm{~km} / \mathrm{s}]$
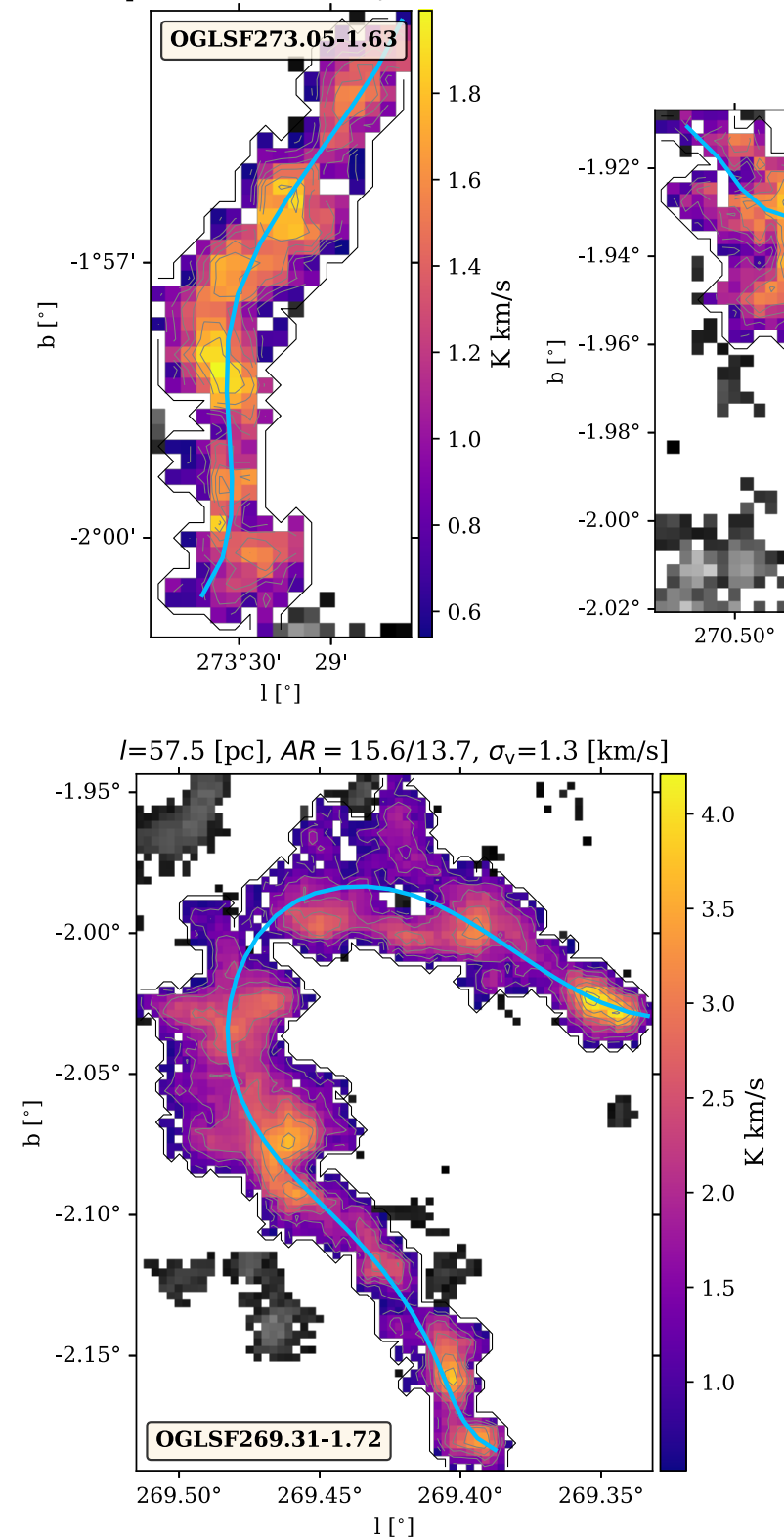

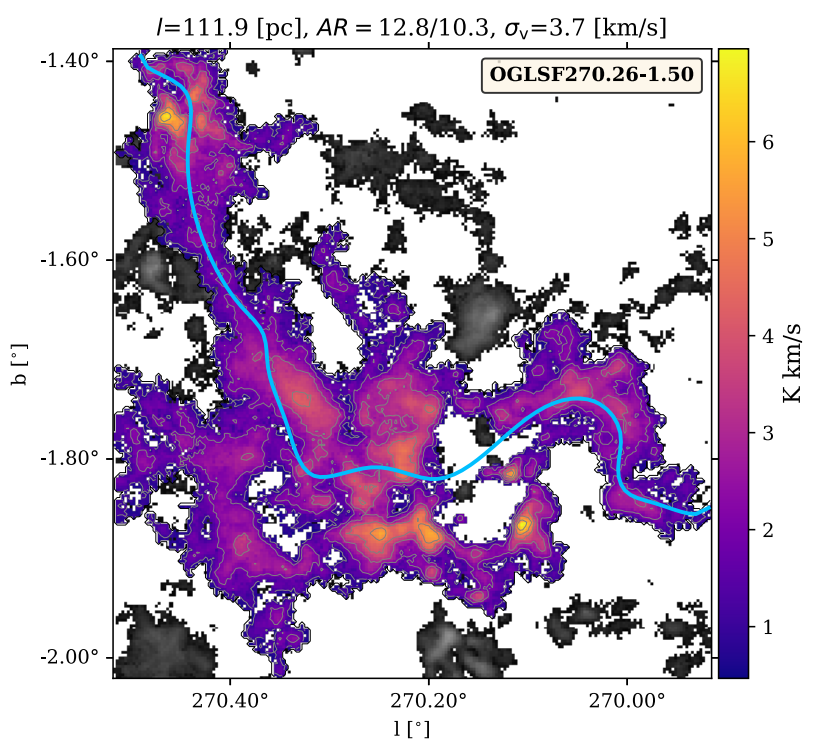

$I=24.1[\mathrm{pc}], A R=10.0 / 8.6, \sigma_{\mathrm{v}}=1.1[\mathrm{~km} / \mathrm{s}]$

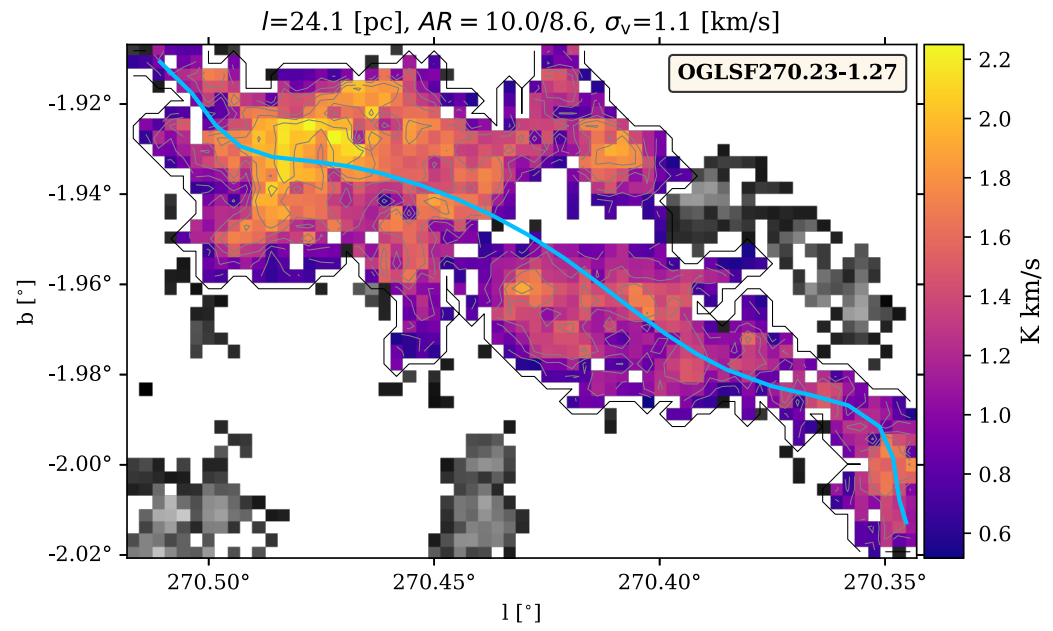

$I=18.7[\mathrm{pc}], A R=9.6 / 11.3, \sigma_{\mathrm{v}}=0.6[\mathrm{~km} / \mathrm{s}]$

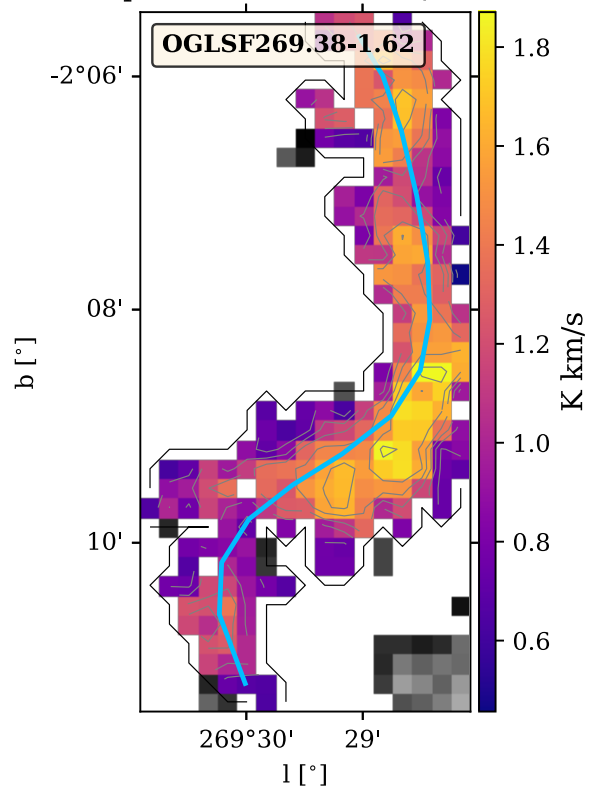

Fig. C.5. Integrated intensity maps of the ${ }^{12} \mathrm{CO}(2-1)$ emission from OGLSFs. Symbols and conventions are the same as in Fig. 1. 